\title{
CONVERGENCE OF A SEQUENCE OF POWERS
}

\author{
R. E. DEMARR ${ }^{1}$
}

A well-known theorem states that if a stochastic matrix (definition below) of finite order has all positive entries in it, then the sequence of its powers (or iterates) converges to a limit; see [3, p. 173]. In this paper we will give a new proof of this result using elementary ideas from the theory of partially ordered linear algebras. Our proof does not use the internal structure of the given matrix; therefore, it can be applied to nonnegative operators.

The basic definition of a partially ordered linear algebra (pola) is as follows. A pola $\mathrm{A}$ is first of all a linear algebra with real numbers as scalars. Real numbers will usually be denoted by small Greek letters. Multiplication of elements of $A$ is assumed to be associative, but not necessarily commutative. Next, the linear algebra $A$ is a partially ordered set subject to the following conditions $(x, y, z$ denote arbitrary elements of $A$ and $\alpha$ denotes an arbitrary real number under the specified restrictions in each condition):

(a) if $x \leqq y$, then $x+z \leqq y+z$;

(b) if $0 \leqq x$ and $0 \leqq y$, then $0 \leqq x y$;

(c) if $0 \leqq \alpha$ and $0 \leqq x$, then $0 \leqq \alpha x$;

(d) for any $x \in A$ there exists $y \geqq 0$ and $z \geqq 0$ such that $x=y-z$.

We may also introduce a form of order completeness described as follows. The pola $A$ is said to be Dedekind $\sigma$-complete if it satisfies the following condition: if $\left\{x_{n}\right\}$ is a sequence of elements from $A$ such that $x_{1} \geqq x_{2} \geqq \cdots \geqq 0$, then $\inf \left\{x_{n}\right\}$ exists. See $[4$, pp. 9-11]. Of course, inf $\left\{x_{n}\right\}$ denotes the infinum (greatest lower bound) of the sequence $\left\{x_{n}\right\}$. It is defined as follows: $\inf \left\{x_{n}\right\}=x$ means that

(1) $x \leqq x_{n}$ for all $n$;

(2) if $y \leqq x_{n}$ for all $n$, then $y \leqq x$.

We now introduce a concept of order convergence: a sequence $\left\{y_{n}\right\}$ of elements from $A$ is said to order converge to $y \in A$ if and only if there exists a sequence $\left\{z_{n}\right\}$ of elements from $A$ such that $z_{1} \geqq z_{2}$ $\geqq \cdots \geqq 0$, inf $\left\{z_{n}\right\}=0$, and $-z_{n} \leqq y_{n}-y \leqq z_{n}$ for all $n$. In this case we write o-lim $y_{n}=y$.

In general, multiplication is not continuous with respect to order convergence; see [2]. We say that multiplication is continuous if the following holds: for every sequence $\left\{x_{n}\right\}$ such that $x_{1} \geqq x_{2} \geqq \cdots \geqq 0$

Received by the editors March 12, 1969.

1 This research was supported in part by the National Science Foundation. 
and $\inf \left\{x_{n}\right\}=0$ and for every $y \geqq 0$ we have $\inf \left\{x_{n} y\right\}=\inf \left\{y x_{n}\right\}=0$.

The reader may find more basic information on partially ordered sets, etc., in [1] and [5].

If $m$ is a fixed positive integer and if $A$ denotes the real linear algebra of all matrices of order $m$ with real entries, then $A$ can be regarded as a pola as follows. If $x \in A$ and $y \in A$, where $x=\left[\alpha_{i j}\right]$ and $y=\left[\beta_{i j}\right]$, than $x \leqq y$ means that $\alpha_{i j} \leqq \beta_{i j}$ for all $i, j$. It is easy to show that in $A$ multiplication is continuous. A stochastic matrix $\left[\alpha_{i j}\right]$ is one such that $\alpha_{i j} \geqq 0$ for all $i, j,=1, \cdots, m$ and $\sum_{i=1}^{m} \alpha_{i j}=1$ for all $i=1$, . . , m. Now suppose $x=\left[\alpha_{i j}\right]$ is a stochastic matrix with $\alpha_{i j} \geqq \delta>0$ for all $i, j$. It is easily seen that $0 \leqq x^{n} \leqq \delta^{-1} x$ for all $n$. It turns out that this is all that is needed to prove that o-lim $x^{n}$ exists. One can easily construct other kinds of nonnegative matrices which satisfy this condition. By referring to [2] the reader will see these ideas can be applied to bounded operators on a real Banach space. We now prove the main theorem.

Theorem. Let $A$ be a partially ordered linear algebra which is Dedekind $\sigma$-complete. If $x \in A$ and if for some $\beta \geqq 1$ we have $0 \leqq x^{n} \leqq \beta x$ for all $n=1,2, \cdots$, then $\mathrm{o}-\lim x^{n}=u$ exists. Also, $0 \leqq u^{2} \leqq u$. If, in addition, we assume that multiplication is continuous, then $u=u^{2}$ $=x u=u x$.

Proof. We begin by defining $\lambda_{1}=\beta$ and then by induction $\lambda_{n+1}$ $=\lambda_{n}\left(1+\lambda_{1}\right)\left(\lambda_{1}+\lambda_{n}\right)^{-1}$ for all $n=1,2, \ldots$. This latter expression can be rewritten $\lambda_{n+1}=1+\lambda_{1}\left(\lambda_{n}-1\right)\left(\lambda_{1}+\lambda_{n}\right)^{-1}$ which means that since $\lambda_{1}$ $=\beta \geqq 1$, we have $\lambda_{n} \geqq 1$ for all $n$. Consequently, we see that $0 \leqq \lambda_{n+1}-1$ $\leqq \lambda_{1}\left(1+\lambda_{1}\right)^{-1}\left(\lambda_{n}-1\right)$ for all $n$. If we put $\alpha=\beta(1+\beta)^{-1}<1$, then we can show by induction that $\lambda_{n}-1 \leqq \alpha^{n-1}(\beta-1)$ for all $n$.

We now show by induction that for each $n$ we have $x^{k} \leqq \lambda_{n} x^{n}$ for all $k \geqq n$. The assumption in our theorem states that this is true if $n=1$. Now suppose that for some $n=p \geqq 1$ we have $x^{k} \leqq \lambda_{p} x^{p}$ for all $k \geqq p$. Take any $q \geqq p$ and define $r=q+1-p \geqq 1$. Now note that 0 $\leqq\left(\lambda_{1} x-x^{r}\right)\left(\lambda_{p} x^{p}-x^{q}\right)$ or $\left(\lambda_{1}+\lambda_{p}\right) x^{q+1} \leqq \lambda_{1} \lambda_{p} x^{p+1}+x^{q+r}$, which is obtained from the previous inequality after multiplying and using the fact that $r+p=q+1$. Now since $q+r-1 \geqq p$, we see that $x^{q+r-1} \leqq \lambda_{p} x^{p}$ which means that $x^{q+r} \leqq \lambda_{p} x^{p+1}$. Therefore,

$$
\left(\lambda_{1}+\lambda_{p}\right) x^{q+1} \leqq\left(\lambda_{1} \lambda_{p}+\lambda_{p}\right) x^{p+1},
$$

which means that $x^{q+1} \leqq \lambda_{p+1} x^{p+1}$ for all $q+1 \geqq p+1$. This completes the proof by induction.

Now let us define $z_{n}=\mu \alpha^{n} x$ and $y_{n}=x^{n}+z_{n}$, where $\mu=(\beta-1)(1+\beta)^{2}$. It is clear that $z_{1} \geqq z_{2} \geqq \cdots \geqq 0$ and $\inf \left\{z_{n}\right\}=0$. We note that 
$z_{n}-z_{n+1}=\alpha^{n-1} \beta(\beta-1) x$, which can easily be computed by recalling that $\alpha=\beta(1+\beta)^{-1}$. Now $0 \leqq \lambda_{n} x^{n}-x^{n+1}=x^{n}-x^{n+1}+\left(\lambda_{n}-1\right) x^{n} \leqq x^{n}$ $-x^{n+1}+\alpha^{n-1}(\beta-1) \beta x=x^{n}-x^{n+1}+z_{n}-z_{n+1}=y_{n}-y_{n+1}$. Consequently, $y_{1} \geqq y_{2} \geqq \cdots \geqq 0$. Since $A$ is Dedekind $\sigma$-complete, we know that $u=\inf \left\{y_{n}\right\}$ exists. It is easy to show that $-z_{n} \leqq x^{n}-u \leqq y_{n}-u$ for all $n$. Since inf $\left\{y_{n}-u+z_{n}\right\}=0$, we have that o-lim $x^{n}=u$.

It is easily seen that $x u \leqq x y_{n}=x^{n+1}+x z_{n} \leqq x^{n+1}+(1+\beta) . z_{n+1}$ $=y_{n+1}+\beta z_{n+1}$ for all $n$. Thus, $x u \leqq u$. From this it follows that $x^{n} u \leqq u$ for all $n$. Hence it follows that $u^{2} \leqq y_{n} u=x^{n} u+z_{n} u \leqq u+\mu \alpha^{n} u$ for all $n$. Hence $u^{2} \leqq u$.

Now let us assume that multiplication is continuous. Since $0 \leqq x y_{n}-x u=x\left(y_{n}-u\right)$ and since $\inf \left\{x\left(y_{n}-u\right)\right\}=0$, we see that $\inf \left\{x y_{n}\right\}=x u$. It is clear that $x y_{n} \geqq x^{n+1}$, which means that $x y_{n}+z_{n}$ $\geqq y_{n+1} \geqq u$ for all $n$. Since $\inf \left\{x y_{n}+z_{n}\right\}=x u$, we see that $x u \geqq u$. We have already shown that $x u \leqq u$. Hence, $x u=u$. Similarly, we can show that $u x=u$.

Now $0 \leqq u y_{n}-u^{2}=u\left(y_{n}-u\right)$. Since $\inf \left\{u\left(y_{n}-u\right)\right\}=0$, we see that $\inf \left\{u y_{n}\right\}=u^{2}$. From what was just proved above we see that $u y_{n}$ $=u+\mu \alpha^{n} u$ for all $n$. Hence, $u^{2}=\inf \left\{u y_{n}\right\}=u$.

\section{REFERENCES}

1. G. Birkhoff, Lattice theory, 3rd ed., Amer. Math. Soc. Colloq. Publ., vol. 25, Amer. Math. Soc., Providence, R. I., 1967.

2. R. E. DeMarr, On partially ordering operator algebras, Canad. J. Math. 19 (1967), 636-643.

3. J. L. Doob, Stochastic processes, Wiley, New York, 1953.

4. E. J. McShane, Order-preserving maps and integration processes, Annals of Math. Studies, no. 31, Princeton Univ. Press, Princeton, N.J., 1953.

5. B. Z. Vulikh, Introduction to the theory of partially ordered spaces, GITTL, Moscow, 1961.

University of New Mexico 